\title{
Mathematical Modeling of the Mixing and Heat Transfer in Turbulent Two-Phase Jets of Mutually Immiscible Liquids
}

\author{
IVAN V. KAZACHKOV \\ Department of Information Technology and Data Analysis \\ Nizhyn Mykola Gogol State University \\ 16600 Grafska str. 2, Nizhyn \\ UKRAINE
}

\begin{abstract}
The paper is devoted to the development and analysis of the mathematical model for mixing and heat transfer in the two-fluid turbulent heterogeneous jet of mutually immiscible liquids. Many natural and technical processes deal with the turbulent jets of mutually immiscible liquids, which represent an important class of the modern multiphase system dynamics. Differential equations for the axially symmetrical twodimensional stationary flow and the integral correlations in a cylindrical coordinate system are considered for the jet of fluid flowing from a nozzle into a pool of another fluid immiscible with the first one. The results may be of interest for researchers and engineers in the multiphase turbulent jets, mixing and heat transfer processes.
\end{abstract}

Key-Words: - Heterogeneous; Turbulent Jet; Mutually Immiscible; Function-Indicator; Two-Phase; Model

Received: February 16, 2020. Revised: July 29, 2020. Accepted: August 4, 2020. Published: August 13, 2020.

\section{Introduction}

The field of turbulent heterogeneous jet flows is now huge. Every month new papers are published. The problem of mathematical modelling and simulation, as well as development of the new experimental techniques is requested by control of turbulent jet flows in a number of diverse technological and technical processes and devices. It has applications in various fields: combustion, aerodynamic noise, propulsion, chemical industry and metallurgy, and many others [1-20].

Control of the mixing intensity of components in the jet flows is performed using the parametric actions, instability processes, etc., e.g. the turbulent mixing of the chemical species in combustors is done not only for enhancing the combustion efficiency, but also for the reduction of the pollutants' emission. The parametric excitation and suppression of the oscillations in a turbulent jet can be significantly altered by applying a suitable excitation at the jet orifice or volumetrically (distributed in space is especially effective) in case of the electroconductive liquid [1]. The external forces interact with the Eigen modes of the oscillations in a jet, mostly in nonlinear way. Also it is available interaction of the modes, and in some cases it is not possible to optimize the process or to increase mixing acting in the resonance modes [1].

Many investigations have been devoted to the gas dynamics of two-phase media, transport features in compressible heterogeneous turbulent jets, peculiarities of high-speed gas-liquid flows, interaction of the phases in multicomponent multiphase jets including accounting the thermal effects, flows with droplets and particles [3-12].

The theoretical and experimental methods were developed to study the structure of turbulent jets, the stability and mixing properties under diverse conditions in the free and confined jet flows, and many other phenomena [13-20]. Not many papers were done concerning the two-phase flows of immiscible fluids like the water and oil [20], which represent specific case of two-phase flows with both deformable liquid phases.

This was a kea point in development of the jet steel making machine proposed by Prof. A.I. Nakorchevskii [21], therefore he developed that method and we were the first performing modelling and computer simulation [22-24]. A number of papers by turbulent heterogeneous jets including the ones mentioned in this paper do not cover this aspect. There are no analogues of the Prof. A.I. Nakorchevskii method [22] by the turbulent heterogeneous jets, where the critical point is to study the detail phase distribution in case of close density ration (e.g. water and oil, metal melt and slag, etc.). Therefore, this paper has a goal to present the results by modelling and computer simulation of the heterogeneous turbulent jets of immiscible liquids with underlining the pros and cons in application of the method, which might be interesting for the other fields of study. 


\section{Statement for Modelling of Multiphase Turbulent Flows}

\subsection{The Method for the Heterogeneous Turbulent Flows}

According to the method by Nakorchevskii [22], all parameters $a^{l}(t)$ (density of liquid, flow velocity, temperature, etc.) of a mixture in the turbulent multiphase flow are considered as follows:

$$
a^{l}(t)=\sum_{i=1}^{m} B_{i}(t) a_{i}^{l}(t), \sum_{i=1}^{m} B_{i}=1 .
$$

The function-indicator $B_{i}(t)$ was introduced for the phases in multiphase flow by the next rule:

$B_{i}(t)= \begin{cases}1, & i \text {-phase occupies elementary volume } \delta \mathrm{V} ; \\ 0, & i \text {-phase outside elementary volume } \delta \mathrm{V} .\end{cases}$

Thus, at each point of the flow there are considered parameters of a flow plus indication of the phase at this point. At each moment of time $B_{i}(t)$ is 1 or 0 for each phase because only one phase can be at the point at the moment of time.

If the function-indicator $B_{i}(t)$ is averaged by time, it has values from 1 to 0 , and the sum of all function-indicators for all phases of a flow is totally 1 (all the time at each point there is present some phase, and only one at the moment). This method was elaborated and applied successfully to solution of a number of different problems [22-28]. The analog of the Navier-Stokes equations in a boundary layer approach was derived in the form [22]:

$$
\begin{gathered}
\sum_{i=1}^{m}\left\lfloor\frac{\partial}{\partial x}\left(y \rho_{i} B_{i} u_{i}\right)+\frac{\partial}{\partial y}\left(y \rho_{i} B_{i} v_{i}\right)\right\rfloor=0, \\
\sum_{i=1}^{m} \rho_{i} B_{i}\left(u_{i} \frac{\partial u_{i}}{\partial x}+v_{i} \frac{\partial u_{i}}{\partial y}\right)=-\frac{d p}{d x}+\frac{1}{y} \frac{\partial}{\partial y}\left\lfloor y \sum_{i=1}^{m} B_{i} \tau_{i}\right\rfloor_{m},
\end{gathered}
$$

where the stationary equations (1) for the flow of incompressible liquids are written in a cylindrical coordinate system. Here are: $p$ - pressure, $\rho$ - density, $u, v$ - the longitudinal and transversal velocity components by $x$ and $y$, respectively, $\tau_{i}-i$-phase turbulent stress.

All parameters of the flow are averaged on the characteristic time interval chosen. Index $m$ belongs to the values at the axis of the flow (symmetry axis). The function-indicator of a phase in multiphase flow may be considered as the mathematical expectation, in contrast to the other multiphase approaches [29, 30], which are based on the introduction of the volumetric specific content of a phase in multiphase flow. Nevertheless, use of the function-indicator allows computing the volumetric specific content of the phases, which have been introduced by another multiphase approaches.

\subsection{Statement for the Model Development}

Development of the model for two-phase jet of immiscible liquids is done according to the Fig. 1 and schematic representation in Fig. 2, where $r_{0}-$ radius of a nozzle, $u_{01}$ - velocity at the nozzle.

The conical surface 1 in Fig. 1 is a boundary of a homogeneous potential core $a$, the internal sublayer $b_{1}$ contains an ejected liquid as a disperse phase, while the external sublayer $b_{2}$ contains the liquid outgoing from the nozzle as a disperse phase. The internal and external sublayers are divided by the surface of phase inverse 2; and the surface 3 is dividing the turbulent and laminar flow zones 3, which is the most indefinite one; 4 is an external conical surface of the axisymmetrical mixing zone (conditionally smooth).

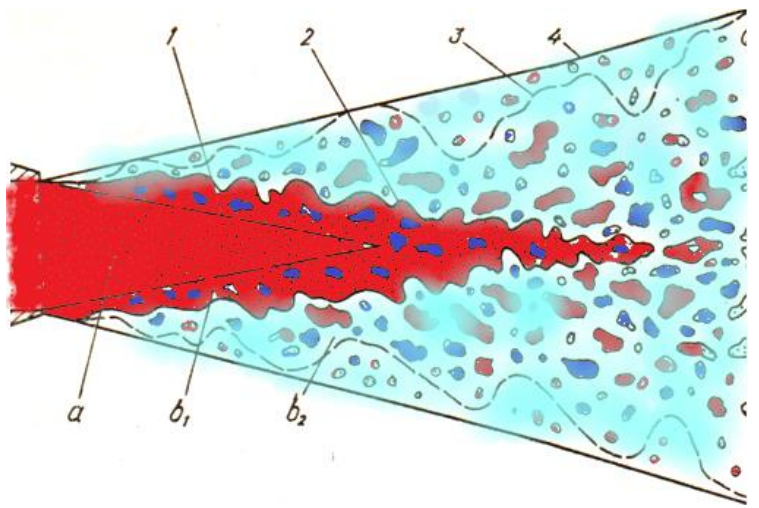

Fig. 1 General view of the multiphase turbulent jet in the pool of other immiscible liquid [22]

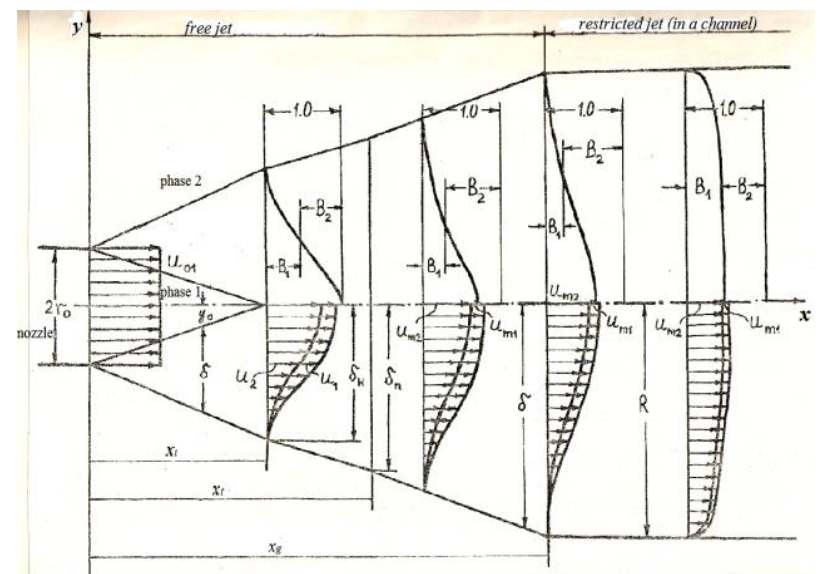

Fig. 2 Schematic representation [22] for multiphase turbulent jet confined by channel at the distance $x_{g}$

Due to a limited regularity of the processes occurring in turbulent jets, the surfaces 2 and 3 in 
Fig. 1 are blurred into the corresponding regions of inversion and intermittency. The external boundary of the jet is the outer envelope surface 4 of the set of surfaces 3 . The uniform velocity profile is assumed for the first liquid going from the nozzle. The surrounding liquid (phase 2) is in the rest before the first liquid starts flowing from the nozzle.

The structural scheme for the mixing process in Fig. 2 is simplified: the initial part of the length $x_{\mathrm{i}}$ with the approximately linear boundaries for the conical surface (in cylindrical coordinate system) of the internal core of a first phase and mixing zone between internal and external boundaries of the jet. The turbulent zone contains fragments of the phases as far as immiscible liquids have behaviors like the separate phases, with their interfacial multiple surfaces. The first phase in a potential core is totally spent in an initial part of the mixing zone. Then a short transit area follows. Afterwards the ground part of the two-phase jet begins, with the two phases well mixed across the entire layer of a jet.

Except for the parameters of phases, the function-indicator of $i$-phase $B_{i}(t)$ shows an influence of $i$-th phase at each point of a space. Normally the spatial averaging of the conservation equations of mass, momentum and energy is performed for a description of multiphase flows based on the concept of volumetric phase content $[29,30]$, which does not fit to the experimental study of a movement of the separate phases in a mixture. In a contrast, an approach [22] with its special experimental technology and a micro sensor for the measurements in two-phase flows fits well for such flows. Actually all known methods of multiphase flows are connected and the parameters averaged by time [22] can be transformed to the ones averaged by space $[29,30]$.

The external boundary of a mixing zone is determined by zero longitudinal velocity of the second phase and zero transversal velocity of the first phase (the second phase is sucked from an immovable surrounding liquid into a mixing zone). The function-indicator of the first phase $B_{1}(t)$ is zero at the external interface because it is absent in the surrounding medium. Similar, the function-indicator $B_{2}(t)$ is zero on the boundary of the potential core, the interface of the first phase flowing from the nozzle. In a first approach, an influence of the mass, viscous and capillary forces is neglected.

With account of the above-mentioned, the boundary conditions are [22]:

$$
\begin{gathered}
y=y_{0}, \quad u_{i}=u_{0 i}, v_{i}=0, \quad \tau_{i}=0, \quad B_{1}=1, \partial B_{1} / \partial \eta=0 \\
y=y_{0}+\delta, \quad u_{i}=0, \quad v_{i}=0, \tau_{i}=0, B_{1}=0 .
\end{gathered}
$$

And dependence of the function-indicator $B_{1}$ from the longitudinal coordinate $x$ is introduced through the second derivative of it at the boundary of a jet:

$$
y=y_{0}, \partial^{2} B_{1} / \partial \eta^{2}=h(x) .
$$

\section{Approximations of the Velocity Profiles and Function-Indicator}

The turbulent stress in the phase is stated by the "new" Prandtl's formula $\tau_{i}=\rho_{i} \kappa_{i} \delta u_{m i} \partial u_{i} / \partial y$, where $\kappa_{i}$ is the coefficient of turbulent mixing for the $i$-th phase, $\delta$ is the width of the mixing layer.

\subsection{Approximations at Initial Part of the Jet}

The polynomial approximations for the velocity profiles and other functions have been obtained based on the boundary conditions (2) [22, 23]. The velocity profiles for the first and second phases are:

$$
\begin{gathered}
u_{1} / u_{01}=1-4 \eta^{3}+3 \eta^{4}, \\
u_{2} / u_{02}=1-6 \eta^{2}+8 \eta^{3}-3 \eta^{4} .
\end{gathered}
$$

\subsection{Approximation of the Function-indicator}

The approximation of the function-indicator was performed with account of the boundary conditions (2), (3):

$$
\begin{gathered}
B_{1}=B_{1}^{(0)}=1-\eta^{3}+0.5 \eta^{2}(1-\eta) h(x), \quad h \in[-6,0] ; \\
B_{1}=B_{1}^{(1)}=1-4 \eta^{3}+3 \eta^{4}+0.5 \eta^{2}(1-\eta)^{2} h(x), \\
h \in[-12,-6] ; \\
B_{1}^{(2)}=1-10 \eta^{3}+15 \eta^{4}-6 \eta^{5}+0.5 \eta^{2}(1-\eta)^{3} h(x), \quad(5) \\
h \in[-20,-12] ; \\
B_{1}^{(3)}=1-20 \eta^{3}+45 \eta^{4}-36 \eta^{5}+10 \eta^{6}+0.5 \eta^{2}(1-\eta)^{4} h(x), \\
h \in[-30,-20] ; \\
B_{1}^{(4)}=1-35 \eta^{3}+105 \eta^{4}-126 \eta^{5}+70 \eta^{6}-15 \eta^{7}+ \\
+0.5 \eta^{2}(1-\eta)^{5} h(x), h \in[-42,-30] ; \\
B_{1}^{(5)}=1-56 \eta^{3}+210 \eta^{4}-336 \eta^{5}+280 \eta^{6}-120 \eta^{7}+ \\
+21 \eta^{8}+0.5 \eta^{2}(1-\eta)^{6} h(x), \quad h \in[-56,-42] ; \\
B_{1}^{(6)}=1-84 \eta^{3}+378 \eta^{4}-756 \eta^{5}+840 \eta^{6}-540 \eta^{7}+ \\
+189 \eta^{8}-28 \eta^{9}+0.5 \eta^{2}(1-\eta)^{7} h(x), \quad h \in[-72,-56] .
\end{gathered}
$$

Here the function $h(x)=\left(\partial^{2} B_{1} / \partial \eta^{2}\right)_{\eta=0}$ describes the variation of $B_{1}$ by $x$, it may vary in the range $h \leq 0$ due to requirements of its physical meaning. The first approximation $B_{1}(\eta)$ in the set (5) reveals 
restricted application in the range $h \in[-6,0]$, while outside of this region it does not satisfy the boundary conditions (2) and the condition $0 \leq B_{1}(\eta) \leq 1, \forall \eta \in[0,1]$. Therefore, all the next approximations $B_{1}(\eta)$ in (5) were obtained as transition of the piecewise continuous functionindicator $B_{1}^{(n)}$ to its next approximation under request that the derivative by $\eta$ with respect to a point $\eta=1$ is zero up to $(n+1)$-th order. These functions have the breaks at the transition points of the permanent characteristic function $B_{1}^{(n)}(\eta, h)$ from the one regional approximation to the other one (the first derivative has a break at those points).

The advantage of such approximations is that all functions $B_{1}^{(n)}$ are smoothly transforming from one region by $h(x)$ to the next one as shown in Fig. 3 . Each function $B_{1}^{(n)}$ exactly coincides with the previous one $B_{1}^{(n-1)}$ at the conjugation boundary, where the $B_{1}^{(n-1)}$ ends while the next, $B_{1}^{(n)}$ starts.

Physical meaning of the varying approximations $B_{1}^{(n)}$ is determined by dependence of the phase distribution in the mixing layer on the density ratio of mixing phases: the higher is density of the surrounding liquid, the shorter is penetration of the first, lighter phase, into the mixing layer.

The disadvantage of the above approximations is a growing complexity of the functions $B_{1}^{(n)}$ in analytical calculation, which is nevertheless easily fought by the modern computer analytical calculations. It is impossible to get a common approximation for $B_{l}(\eta, h)$ satisfying the boundary conditions (2) in all range by parameter $i_{0}$ (due to the requirement of variation $B_{1}$ from 0 to 1 ).

\subsection{Approximations at Ground Part of Jet}

The polynomial approximations for $u_{2}, B_{1}$ remain the same on a ground part of the jet but for the function $u_{l}$ the approximation is

$$
u_{1} / u_{m 1}=1-3 \eta^{2}+2 \eta^{3} \text {. }
$$

\subsection{Calculation of the Profiles}

All polynomial approximations (3)-(6) are shown in the Figs 3, 4:

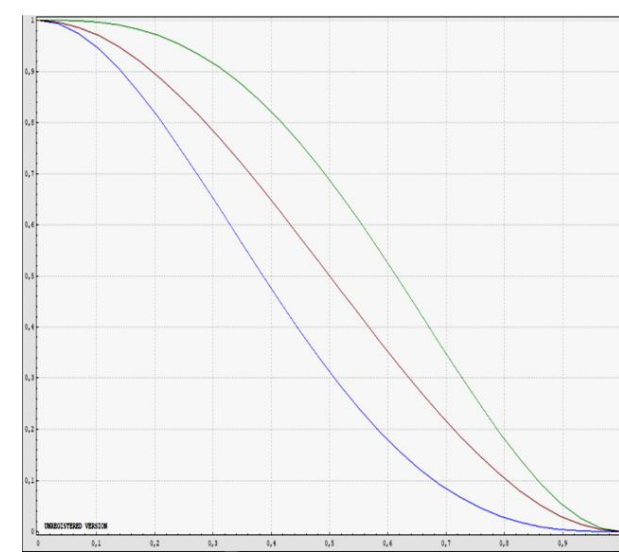

Fig. 3 Velocity profiles across the layer (from top to bottom): $1-u_{1} / u_{01}, 2-u_{1} / u_{m 1}, 3-u_{2} / u_{02}$

The real profiles for the multiphase flow are represented as a product of the corresponding profile multiplied by its function-indicator. The ones are presented in Figs 5, 6 for the few regions by the function $h(x)$.

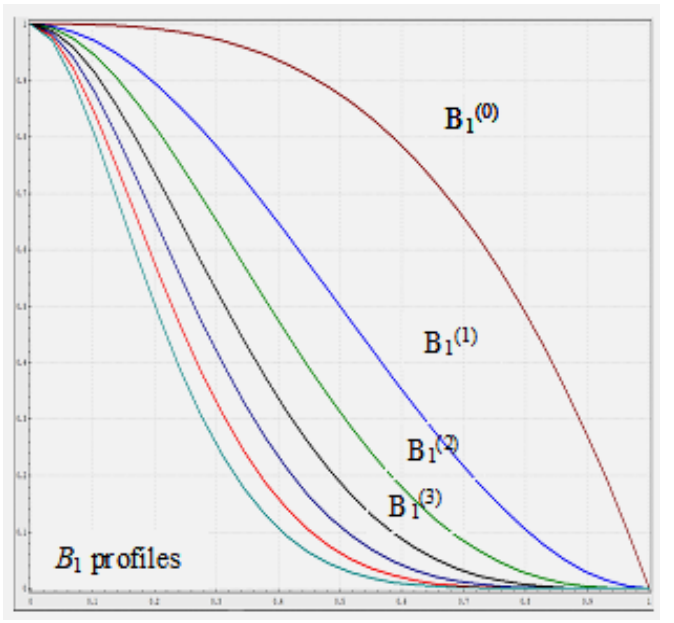

Fig. 4 Approximations of the profiles of functionindicator $B_{1}^{(n)}$ across the layer

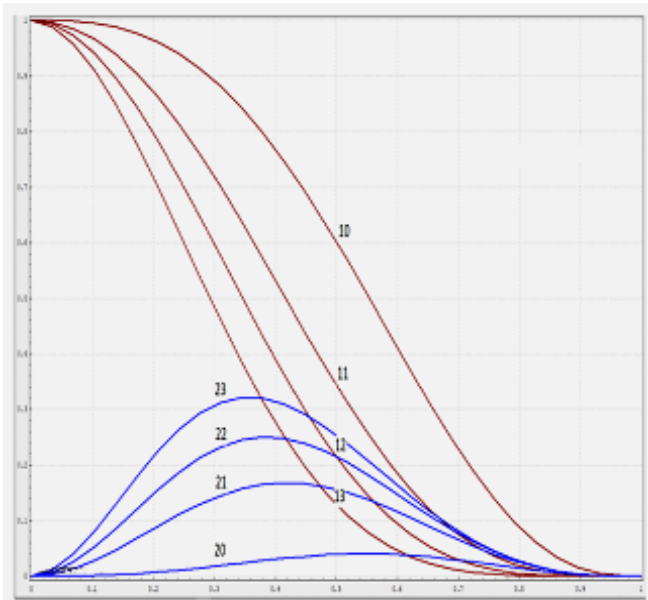

Fig. 5 Real velocity profiles of first and second phase for $h(x): 0,-6,-12,-20$ at the initial part of jet, 10-13: $B_{1} u_{1} / u_{01}, 20-23: B_{2} u_{2} / u_{02}$ 


\section{Integral Correlations for the Initial and Ground Parts of the Jet}

Based on the above approximations of the profiles (3)-(5), the integral correlations have been derived for the two-phase turbulent jet [22, 23].

\subsection{The Initial Part of the Jet}

Integration of the mass and momentum conservation equations (1) with the boundary conditions (2) for the total cross-section of a flow $y=y_{0}+\delta$, as well as the momentum conservation equation for the part $y=y^{*}$ of the jet, respectively, yields the equation array (7).

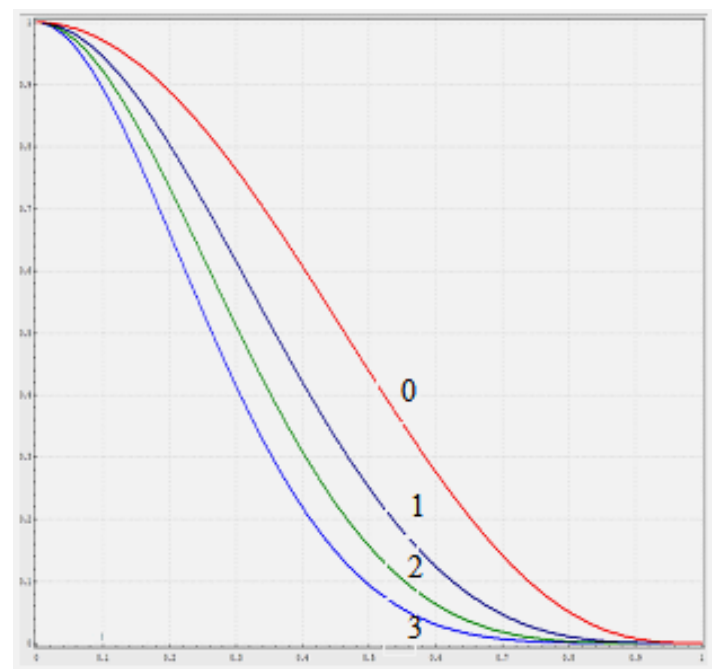

Fig. 6 The real velocity profiles of the first phase at the ground part of jet for $h(x): 0,-6,-12,-20$;

$$
\begin{gathered}
0-3: B_{1} u_{1} / u_{m 1} \\
u_{01}\left(r_{0}^{2}-y_{0}^{2}\right)=2 \delta \int_{0}^{1} B_{1} u_{1}\left(y_{0}+\delta \eta\right) d \eta \\
\rho_{1} u_{01}^{2}\left(r_{0}^{2}-y_{0}^{2}\right)=2 \delta \int_{0}^{1}\left(\rho_{1} B_{1} u_{1}^{2}+\rho_{2} B_{2} u_{2}^{2}\right) . \\
\cdot\left(y_{0}+\delta \eta\right) d \eta, \quad \rho_{1} u_{01}\left(u_{01}-u_{1}^{*}\right) \frac{d y_{0}}{d x}+ \\
+\frac{d}{d x} \delta \int_{0}^{\eta} \sum_{j=1}^{*} \rho_{j} B_{j} u_{j}^{2}\left(y_{0}+\delta \eta\right) d \eta= \\
=\sum_{j=1}^{2} u_{i}^{*} \frac{d}{d x} \delta \int_{0}^{\eta^{*}} \rho_{i} B_{i} u_{i}\left(y_{0}+\delta \eta\right) d \eta+ \\
+\left(y_{0}+\delta \eta^{*}\right) \sum_{j=1}^{2} \rho_{j} B_{j} \kappa_{j} u_{0 j} \frac{\partial u_{j}^{*}}{\partial \eta}, \quad B_{1}+B_{2}=1 .
\end{gathered}
$$

The first equation (7) was got integrating the mass conservation equation by $y$, the second and the third ones - integrating the momentum conservation for the total flow of a two-phase mixture for $y=y_{0}+\delta$ and $y=y^{*}$, respectively. The parameters at $\eta=\eta^{*}<1$ are signed with a star*.

\subsection{The Ground Part of the Jet}

The integral correlations for the ground part of a jet are [22]:

$$
\begin{gathered}
\int_{0}^{\delta} B_{1} u_{1} y d y=\frac{u_{01} r_{0}^{2}}{2}, \sum_{j=1}^{2} \int_{0}^{\delta} \rho_{j} B_{j} u_{j}^{2} y d y=\rho_{1} \frac{u_{01}^{2} r_{0}^{2}}{2} \\
\frac{d}{d x} \sum_{j=1}^{2} \int_{0}^{y^{*}} \rho_{j} B_{j} u_{j}^{2} y d y=y^{*} \sum_{j=1}^{2} B_{j}^{*} \tau_{j}^{*}+ \\
+\sum_{j=1}^{2} u_{j}^{*} \frac{d}{d x} \int_{0}^{y^{*}} \rho_{j} B_{j} u_{j} y d y
\end{gathered}
$$

where the first is equation of mass conservation for the first phase, the second and the third - the momentum conservation equations for the total and for the part of the cross section, respectively, according to the methodology [31].

The integral correlations for the ground part of a jet were obtained similarly to the initial part. The momentum equations for the total and for the part of the cross section, respectively, were got according to [31]. The momentum equation on the jet's axis $(y=0)$ is used too:

$$
\sum_{j=1}^{2} \rho_{j} B_{m j} u_{m j} \frac{d u_{m j}}{d x}=2 \sum_{j=1}^{2}\left\lfloor\frac{\partial}{\partial y}\left(B_{j} \tau_{j}\right)\right\rfloor_{m} .
$$

Mathematical model (7)-(9) including the ordinary differential equations by longitudinal coordinate $x$ are used for analysis and numerical simulation of the basic features of the stationary turbulent two-phase jet of two immiscible liquids.

The function-indicator $B_{1}$ shows how much is a presence of the first phase in a selected point of mixing zone, which can be directly compared to an experimental data by two-phase sensor [22]. Therefore, a solution of the task may give both parameters of the flow together with their belonging to a particular phase.

\subsection{Transformation of the Mathematical Model to the Dimensionless Form}

The equation array (7) for the initial part of the jet is transformed to the following dimensionless form with the scales $r_{0}, \delta, u_{0 i}$ for the longitudinal and transversal coordinates and velocity, respectively:

$$
y_{0}^{2}+2 \delta \sum_{j=1}^{2} y_{0}^{2-j} \delta^{j-1}\left(a_{j+2}+i_{0} b_{j+2}\right)=1,
$$




$$
\begin{gathered}
y_{0}^{2}+2 \delta \sum_{j=1}^{2} y_{0}^{2-j} \delta^{j-1} a_{j}=1 \\
\left(1-u_{1}^{*}\right) y_{0} \frac{d y_{0}}{d \varsigma}-\frac{d}{d \varsigma} \delta \sum_{j=1}^{2} y_{0}^{2-j} \delta^{j-1}\left(a_{j}^{*} u_{1}^{*}+i_{0} b_{j}^{*} u_{2}^{*}\right)+ \\
+\frac{d}{d \varsigma} \delta \sum_{j=1}^{2} y_{0}^{2-j} \delta^{j-1}\left(a_{j+2}^{*}+i_{0} b_{j+2}^{*}\right)= \\
=\left(y_{0}+\delta \eta^{*}\right) \sum_{j=1}^{2} B_{j}^{*}\left(\frac{\partial u_{j}}{\partial \eta}\right)^{*}\left(i_{0} \kappa_{21}\right)^{j-1}
\end{gathered}
$$

Here are the star marked values taken by $\eta=\eta^{*}$. Normally $\eta^{*}=0.5$ is adopted for this. The other assignments are as follows:

$$
\begin{gathered}
\bar{y}_{0}=y_{0} / r_{0}, \bar{\delta}=\delta / r_{0}, \eta=\left(y-y_{0}\right) / \delta, \bar{x}=x / r_{0}, \\
\varsigma=\kappa_{1} \bar{x}, s_{0}=u_{02} / u_{01}, i_{0}=n s_{0}^{2}, b_{i}=b_{i 1}+b_{i 2} h, \\
n=\rho_{2} / \rho_{1}, \kappa_{21}=\kappa_{2} / \kappa_{1}, a_{i}=a_{i 1}+a_{i 2} h, \quad(11) \\
a_{i}=\int_{0}^{1} B_{1} \bar{u}_{1} \eta^{j-1} d \eta, b_{i}=\int_{0}^{1} B_{2} \bar{u}_{2} \eta^{j-1} d \eta(i=1,2) ; j=1,2 \\
a_{i}=\int_{0}^{1} B_{1} \bar{u}_{1}^{2} \eta^{j-1} d \eta, b_{i}=\int_{0}^{1} B_{2} \bar{u}_{2}^{2} \eta^{j-1} d \eta(i=3,4) .
\end{gathered}
$$

\subsubsection{Calculation of Parameters in the Model}

The computed values of the integral parameters in (11) have been done in a range of variation of the function $h(x)$ according to the approximation of the velocity profiles (3), (4) and the function-indicator of the phase $B_{1}(5)$. As shown below mostly the region by parameter $h(x)$ is covered in (5) for the bright enough density ratio of the mixing liquid phases. The coefficients $a_{i j}, b_{i j}$ according to (11),

(3)-(5) are presented in the Table 1 and Table 2:

Table 1 - Integral parameters $a_{i j}$ of the model for different regions of function-indicator $B_{1}$

\begin{tabular}{|c|c|c|c|c|}
\hline $\begin{array}{c}a_{i j} \text { for } h \\
\text { interval: }\end{array}$ & $a_{11}$ & $a_{12}$ & $a_{21}$ & $a_{22}$ \\
\hline $0,-6$ & 0.5464 & 0.0208 & 0.1667 & 0.0101 \\
\hline$-6,-12$ & 0.4857 & 0.0107 & 0.1333 & 0.0046 \\
\hline$-12,-20$ & 0.4310 & 0.0062 & 0.1065 & 0.0023 \\
\hline$-20,-30$ & 0.3844 & 0.0038 & 0.0859 & 0.0013 \\
\hline$-30,-42$ & 0.3455 & 0.0025 & 0.0703 & 0.00078 \\
\hline$-42,-56$ & 0.3128 & 0.0017 & 0.0583 & 0.00049 \\
\hline$-56,-72$ & 0.2853 & 0.0013 & 0.0490 & 0.00032 \\
\hline $\begin{array}{c}a_{i j} \text { for } h \\
\text { interval: }\end{array}$ & $a_{31}$ & $a_{32}$ & $a_{41}$ & $a_{42}$ \\
\hline $0,-6$ & 0.4604 & 0.0139 & 0.1198 & 0.0059 \\
\hline$-6,-12$ & 0.4250 & 0.0080 & 0.1027 & 0.0030 \\
\hline$-12,-20$ & 0.3884 & 0.0049 & 0.0866 & 0.0017 \\
\hline
\end{tabular}

\begin{tabular}{|c|c|c|c|c|}
\hline$-20,-30$ & 0.3542 & 0.0032 & 0.0728 & 0.0010 \\
\hline$-30,-42$ & 0.3237 & 0.0022 & 0.0614 & 0.00064 \\
\hline$-42,-56$ & 0.2969 & 0.0016 & 0.0522 & 0.00042 \\
\hline$-56,-72$ & 0.2734 & 0.0012 & 0.0447 & 0.00028 \\
\hline
\end{tabular}

Table 2 - Integral parameters $b_{i j}$ of the model for different regions of the function-indicator $B_{1}$

\begin{tabular}{|c|c|c|c|c|}
\hline $\begin{array}{r}a_{i j}^{*} \text { for } h \\
\text { interval: }\end{array}$ & $a_{11}{ }^{*}$ & $a_{12}{ }^{*}$ & $a_{21}{ }^{*}$ & $a_{22}{ }^{*}$ \\
\hline $0,-6$ & 0.444 & 0.011 & 0.1029 & 0.0038 \\
\hline$-6,-12$ & 0.421 & 0.007 & 0.0941 & 0.0024 \\
\hline$-12,-20$ & 0.392 & 0.006 & 0.0836 & 0.0015 \\
\hline$-20,-30$ & 0.362 & 0.003 & 0.0730 & 0.00097 \\
\hline$-30,-42$ & 0.333 & 0.002 & 0.0631 & 0.00064 \\
\hline$-42,-56$ & 0.307 & 0.002 & 0.0548 & 0.00043 \\
\hline$-56,-72$ & 0.281 & 0.001 & 0.0468 & 0.00030 \\
\hline $\begin{array}{r}a_{i j}^{*} \text { for } h \\
\text { interval: }\end{array}$ & $a_{31}{ }^{*}$ & $a_{32}{ }^{*}$ & $a_{41}{ }^{*}$ & $a_{42}{ }^{*}$ \\
\hline $0,-6$ & 0.4103 & 0.0094 & 0.0902 & 0.0032 \\
\hline$-6,-12$ & 0.3912 & 0.0062 & 0.0831 & 0.0020 \\
\hline$-12,-20$ & 0.3673 & 0.0042 & 0.0745 & 0.0012 \\
\hline$-20,-30$ & 0.3416 & 0.0029 & 0.0657 & 0.0008 \\
\hline$-30,-42$ & 0.3164 & 0.0021 & 0.0574 & 0.0006 \\
\hline
\end{tabular}

\begin{tabular}{|c|c|c|c|c|}
\hline $\begin{array}{c}b_{i j} \text { for } h \\
\text { interval: }\end{array}$ & $b_{11}$ & $b_{12}$ & $b_{21}$ & $b_{22}$ \\
\hline $0,-6$ & 0.0179 & -0.0101 & 0.0095 & -0.0042 \\
\hline$-6,-12$ & 0.0429 & -0.0060 & 0.0214 & -0.0022 \\
\hline$-12,-20$ & 0.0690 & -0.0038 & 0.0327 & -0.0012 \\
\hline$-20,-30$ & 0.0939 & -0.0025 & 0.0424 & -0.00076 \\
\hline$-30,-42$ & 0.1167 & -0.0018 & 0.0506 & -0.00049 \\
\hline$-42,-56$ & 0.1371 & -0.0013 & 0.0573 & -0.00032 \\
\hline$-56,-72$ & 0.1552 & -0.001 & 0.0629 & -0.00022 \\
\hline $\begin{array}{c}b_{i j} \text { for } h \\
\text { interval: }\end{array}$ & $b_{31}$ & $b_{32}$ & $b_{41}$ & \\
\hline $0,-6$ & 0.0054 & -0.0048 & 0.0023 & -0.0016 \\
\hline$-6,-12$ & 0.0149 & -0.0032 & 0.0059 & -0.00097 \\
\hline$-12,-20$ & 0.0625 & -0.0023 & 0.0098 & -0.00062 \\
\hline$-20,-30$ & 0.0390 & -0.0016 & 0.0141 & -0.00042 \\
\hline$-30,-42$ & 0.0515 & -0.0012 & 0.0180 & -0.00029 \\
\hline$-42,-56$ & 0.0637 & -0.001 & 0.0215 & -0.00021 \\
\hline$-56,-72$ & 0.0752 & -0.0007 & 0.0246 & -0.00015 \\
\hline
\end{tabular}

The integral correlation for the part of the mixing layer in the system (10) was considered at $\eta=0.5$ according to the widely accepted methodology [31]. Corresponding coefficients $a_{i j}^{*}, b_{i j}^{*}$ for the middle section of mixing layer are given in the Tables 3, 4:

Table 3 - Integral parameters $a_{i j}^{*}$ of the model for different regions of the function-indicator $B_{1}$ 


\begin{tabular}{|l|l|l|l|l|}
\hline$-42,-56$ & 0.2936 & 0.0015 & 0.0503 & 0.0004 \\
\hline$-56,-72$ & 0.2711 & 0.0011 & 0.0434 & 0.0003 \\
\hline
\end{tabular}

Table 4 - Integral parameters $b_{i j}^{*}$ of the model for different regions of the function-indicator $B_{1}$

\begin{tabular}{|c|c|c|c|c|}
\hline $\begin{array}{c}b_{i j}{ }^{*} \text { for } h \\
\text { interval: }\end{array}$ & $b_{11}{ }^{*}$ & $b_{12}{ }^{*}$ & $b_{21}{ }^{*}$ & $b_{22}{ }^{*}$ \\
\hline $0,-6$ & 0.0075 & -0.0142 & 0.0028 & -0.0047 \\
\hline$-6,-12$ & 0.0214 & -0.0048 & 0.0079 & -0.0015 \\
\hline$-12,-20$ & 0.0392 & -0.0033 & 0.0141 & -0.0001 \\
\hline$-20,-30$ & 0.0585 & -0.0023 & 0.0206 & -0.0006 \\
\hline$-30,-42$ & 0.0777 & -0.0017 & 0.0267 & -0.0004 \\
\hline$-42,-56$ & 0.0954 & -0.0012 & 0.0320 & -0.0003 \\
\hline$-56,-72$ & 0.1130 & -0.001 & 0.0372 & -0.0002 \\
\hline $\begin{array}{c}b_{i j} \text { for } h \\
\text { interval: }\end{array}$ & $b_{31}{ }^{*}$ & $b_{32}{ }^{*}$ & $b_{41}{ }^{*}$ & \\
\hline $0,-6$ & 0.0039 & -0.0085 & 0.0014 & -0.0025 \\
\hline$-6,-12$ & 0.0114 & -0.0030 & 0.0039 & -0.0008 \\
\hline$-12,-20$ & 0.0214 & -0.0022 & 0.0071 & -0.0006 \\
\hline$-20,-30$ & 0.0328 & -0.0016 & 0.0106 & -0.0004 \\
\hline$-30,-42$ & 0.0446 & -0.0012 & 0.0140 & -0.0003 \\
\hline$-42,-56$ & 0.0560 & -0.0009 & 0.0172 & -0.0002 \\
\hline$-56,-72$ & 0.0675 & -0.0007 & 0.0202 & - \\
& & & & 0.00015 \\
\hline
\end{tabular}

Using the above presented in the tables 1-4 integral parameters of the jet at its initial part, it is available to compute the characteristics of the jet.

Except the above, we retain the same notations for the dimensionless parameters as for the dimensional ones. Only here in (11) it is stated for clarification of the dimensionless notations.

\subsubsection{Statement of the Boundary Conditions}

The sliding factor $s_{0}$ is supposed to be constant. Boundary conditions (2) for (11) are transformed as

$$
\zeta=0, y_{0}=1, \delta=0 ; \quad \zeta=\zeta_{i}, y_{0}=0, \delta=\delta_{i} ;
$$

where $\zeta_{i}, \delta_{i}$ are the dimensionless length of a jet and its maximal radius (at the end of the initial part).

\section{Models for the Initial and Ground Parts of a Jet}

\subsection{Characteristics of the Initial Part of a Jet}

The characteristics $y_{0}(\zeta), \delta(\zeta), h(\zeta)$ are computed from numerical solution of the boundary problem (10), (12). Then all the others are got for the stated values of the main parameters: $i_{0}, \kappa_{1}, \kappa_{2}$. The first parameter is slightly indefinite due to difficulties with estimation of the phases' sliding, while the other two are known from the experimental studies for the specific conditions.

The main problem with validation of the mathematical model against the experimental data is correct estimation of the coefficients of turbulent mixing $\kappa_{1}, \kappa_{2}$ in each specific case. But the advantage of the model is a possibility to have all characteristics of a flow together with their belonging to a particular phase through the functions $B_{1}, B_{2}$. Distribution of the transversal velocities, interface interactions, coefficients of the volumetric $q$ and mass ejection $g$, and kinetic energy $e_{i}$ for the phases in a flow are computed as [22]:

$$
\begin{gathered}
q=2 s_{0} \delta\left(b_{1} y_{0}+b_{2} \delta\right), \frac{B_{1} v_{1}}{\kappa_{1} u_{01}}=B_{1} u_{1} \frac{d}{d \varsigma}\left(y_{0}+\delta \eta\right)+ \\
-\frac{1}{y_{0}+\delta \eta} \frac{d}{d \varsigma}\left(0.5 y_{0}^{2}+a_{1} y_{0} \delta+a_{2} \delta^{2}\right), \\
\frac{B_{2} v_{2}}{\kappa_{1} u_{02}}=B_{2} u_{2} \frac{d}{d \varsigma}\left(y_{0}+\delta \eta\right)+ \\
-\frac{1}{y_{0}+\delta \eta} \frac{d}{d \varsigma}\left(b_{1} y_{0} \delta+b_{2} \delta^{2}\right), \\
e_{1}=y_{0}^{2}+2 \int_{0}^{1} B_{1} \bar{u}_{1}^{3}\left(y_{0}+\delta \eta\right) \delta d \eta, \\
e_{2}=2 i_{0} s_{0} \int_{0}^{1} B_{2} \bar{u}_{2}^{3}\left(y_{0}+\delta \eta\right) \delta d \eta, \\
-1 \\
R_{21}=\frac{\partial u_{1}}{\left(y_{0}+\delta \eta\right) \delta} \frac{d}{\partial \eta}\left(0.5 y_{0}^{2}+a_{1} y_{0} \delta+a_{2} \delta^{2}\right)+ \\
\left.+\frac{\partial}{\partial \eta}\left\lfloor\left(y_{0}+\delta \eta\right) B_{1} \frac{\partial u_{1}}{\partial \eta}\right\rfloor\right\} .
\end{gathered}
$$

\subsection{Equations and Boundary Conditions of the Ground Part of a Jet}

For the short transient part of the jet there is no developed substantiated scheme, therefore it is not under consideration here. The ground part is considered after a short transient part. The method is well elaborated and supported with the experimental data $[22,25,28]$. The dimensionless equation array (8), (9) for the ground part is the next

$$
\begin{gathered}
2 B_{m 1} u_{m 1} \delta^{2} \sum_{j=1}^{2} \alpha_{1 j} h^{j-1}=1 \\
2 B_{m 1} u_{m 1} \delta^{2} \sum_{j=1}^{2}\left(\alpha_{2 j}+i_{0} \beta_{2 j}\right) h^{j-1}+i_{0} \beta_{20}=1
\end{gathered}
$$


$\left.h^{j-1}+i_{0} \beta_{20}^{*}\right\rfloor-u_{m 1} \frac{d}{d \varsigma}\left(u_{m 1} \delta^{2}\right)\left\lfloor B_{m 1} \sum_{j=1}^{2}\left(\alpha_{1 j}^{*} u_{1}^{*}+i_{0} \beta_{1 j}^{*} u_{2}^{*}\right) h^{j-1}+i_{0} u_{2}^{*} \beta_{10}^{*}\right\rfloor=$

$$
=\eta^{*} \delta u_{m 1}^{2}\left[\left(1-i_{0} \kappa_{21}\right) B_{m 1} \sum_{j=1}^{2}\left(\frac{\partial u_{j}}{\partial \eta}\right)^{*} \gamma_{j}^{*} h^{j-1}+i_{0} \kappa_{21}\left(\frac{\partial u_{2}}{\partial \eta}\right)^{*}\right\rfloor .
$$

Here are:

$$
\begin{gathered}
\bar{x}=\frac{x-x_{t}}{r_{0}}, \quad \bar{u}_{m i}=\frac{u_{m i}}{u_{0 i}}, \bar{u}_{i}=\frac{u_{i}}{u_{m i}}, i_{0}=n s_{0}^{2}, \quad \bar{B}_{2}=\frac{B_{2}}{B_{m 1}}, \\
\bar{B}_{1}=\frac{B_{1}}{B_{m 1}}=\gamma_{1}+\gamma_{2} h, \int_{0}^{1} \bar{B}_{1} \bar{u}_{1}^{i} \eta d \eta=\sum_{j=1}^{2} \alpha_{i j} h^{j-1}, \\
\int_{0}^{1} \bar{B}_{2} \bar{u}_{2}^{i} \eta d \eta=\frac{\beta_{i 0}}{B_{m 1}}+\beta_{i 1}+\beta_{i 2} h \quad(i=1,2) .
\end{gathered}
$$

The same as previously, we use these notations for the dimensionless values only here and keep previous assignments for the dimensionless parameters as for the dimension ones in all the other equations. Star * means a value by $\eta=\eta^{*}<1, x_{t}$ is the length of a transient part of a jet flow. It is assumed $u_{\mathrm{m} 2}=s_{0} u_{\mathrm{m} 1} \quad\left(s_{0}=\right.$ const $)$, which means that sliding of the phases is preserved the same as for the initial part of a jet. The boundary conditions for the equation array (14) are

$$
\begin{gathered}
\zeta=0, \quad u_{m l}=1, \quad B_{m l}=1, \quad \delta=\delta_{t} ; \\
\zeta=\infty, \quad u_{m l}=0, \quad B_{m l}=0, \quad \delta=\infty ;
\end{gathered}
$$

$\delta_{t}$ is a radius of the jet at the transient cross section. Solution of the boundary problem (14), (16) yields the functions $u_{m I}(\zeta), B_{m l}(\zeta), \delta(\zeta)$ and $h(\zeta)$ for the stated values $i_{0}, \kappa_{21}$. Then all parameters of a twophase flow are got: turbulent stresses, mass flow rate, trajectories of the phases in a mixing layer.

\section{Solutions of Boundary Problems for the Initial and Ground Part of Jet}

\subsection{Analytical Solution at the Initial Part}

Solution of the boundary problem (10), (12) allows calculating the parameters for the initial part of a jet by the stated values $i_{0}, \kappa_{21}$. Computational experiments revealed the basic features of the flow. The velocity distributions for the phases $\left(u_{1}, u_{2}\right)$ and function-indicators of the phases $\left(B_{1}, B_{2}\right)$ are stated, as well as the parameters at the cross section.
The system (10) contains two algebraic and one differential equation. From the algebraic equation array, the functions $y_{0}(h), \delta(h)$ are got. Then the differential equation is expressed in a standard form $d h / d \zeta=\mathrm{F}\left(h(\zeta), i_{0}, \kappa_{21}\right)$, for the numerical solution. The equation array (10) can be solved numerically in general but the way we applied here is more comprehensive for understanding the basic features of the system, with as much as possible analytical expressions showing the explicit functions.

The variation range of $h(\zeta)$ is determined by substitution of the boundary conditions to the functions $y_{0}(h), \delta(h)$, so that it yields the next:

$$
\begin{gathered}
y_{0}=\frac{1}{\sqrt{1+2 a_{1} \frac{a_{3}+i_{0} b_{3}-a_{1}}{a_{2}-i_{0} b_{4}-a_{4}}+2 a_{2}\left(\frac{a_{3}+i_{0} b_{3}-a_{1}}{a_{2}-i_{0} b_{4}-a_{4}}\right)^{2}}} \\
\delta=y_{0} \frac{a_{3}+i_{0} b_{3}-a_{1}}{a_{2}-i_{0} b_{4}-a_{4}} \\
h_{0}=h(0)=\frac{a_{11}-a_{31}-i_{0} b_{31}}{a_{32}-a_{12}+i_{0} b_{32}} \\
h_{i}=h\left(\varsigma_{i}\right)=\frac{a_{21}-a_{41}-i_{0} b_{41}}{a_{42}-a_{22}+i_{0} b_{42}}
\end{gathered}
$$

The limit values of the $h_{0}$ and $h_{i}$ in (19) are computed from the equations $a_{3}+i_{0} b_{3}-a_{1}=0$, $a_{2}-i_{0} b_{4}-a_{4}=0$ corresponding to the beginning and the end of the initial part of a jet. Here according to the boundary conditions (12), for the functions $y_{0}(\varsigma)$ and $\delta(\varsigma)$ must be $y_{0}(0)=1$, $\delta(0)=0$, and $y_{0}\left(\varsigma_{i}\right)=0, \delta\left(\varsigma_{i}\right)=\delta_{i}$ for any values of the parameters including the parameter $i_{0}$. The first one is satisfied by

$$
A=\frac{a_{3}+i_{0} b_{3}-a_{1}}{a_{2}-i_{0} b_{4}-a_{4}}=0, \Rightarrow a_{3}+i_{0} b_{3}-a_{1}=0,
$$

where from the first correlation (19) follows. The other boundary condition (at the end of initial part) requires $y_{0}\left(\varsigma_{i}\right)=0, A=\infty$, which yields $a_{2}-i_{0} b_{4}-a_{4}=0$ :

$$
\delta_{i}=\lim _{A \rightarrow \infty} \frac{A}{\sqrt{1+2 a_{1} A+2 a_{2} A^{2}}}=\frac{1}{\sqrt{2\left(a_{21}+a_{22} h_{i}\right)}} .
$$

The function $y_{0}(h)$ must be real by any parameters, thus, the value under square root in (18) must be always positive. Therefore, the following requirement is got for the parameters of the approximations available for the use here: $1+2 a_{1} A+2 a_{2} A^{2}>0$, which is satisfied by any $A$ $(A>0)$ in the ranges $A>A_{2}<0, A<A_{1}<0$, and 
$A_{2}=\left(\sqrt{D_{a}}-a_{1}\right) /\left(2 a_{2}\right), A_{1}=-\left(\sqrt{D_{a}}+a_{1}\right) /\left(2 a_{2}\right)$ where $D_{a}<a_{1}, \quad a_{1}>0, \quad a_{2}>0 . \quad$ Because $D_{a}=a_{1}^{2}-2 a_{2}$, from $D_{a} \geq 0$ follows $a_{1}^{2} \geq 2 a_{2}$, or

$$
\left(\int_{0}^{1} B_{1} \bar{u}_{1} d \eta\right)^{2} \geq 2 \int_{0}^{1} B_{1} \bar{u}_{1} \eta d \eta
$$

In our approximations, the values, $a_{1}$ and $2 a_{2}$, are very close, therefore $A_{2} \approx A_{1} \approx-a_{1} /\left(2 a_{2}\right)$ is critical point, where the solution is ill-fated.

Accounting the (11), the inequality (20) yields the following requirement for the function $h$ :

$$
a_{12}^{2} h^{2}+2\left(a_{11} a_{12}-a_{22}\right) h+a_{11}^{2}-2 a_{21} \geq 0,
$$

satisfying strictly $\left(a_{12}^{2}>0\right)$, and the equation $a_{12}^{2} h^{2}+2\left(a_{11} a_{12}-a_{22}\right) h+a_{11}^{2}-2 a_{21}=0$ has no real roots

$$
h_{1,2}=\frac{a_{22}-a_{11} a_{12} \pm \sqrt{D_{h}}}{a_{12}^{2}},
$$

because the discriminant $D_{h}=a_{22}\left(a_{22}-2 a_{11} a_{12}\right)<0$ by our approximations as shown below for the data presented in the Table 1:

$$
\begin{gathered}
D_{h}=-1.3 \cdot 10^{-4}, D_{h}=-2.7 \cdot 10^{-5}, D_{h}=-7 \cdot 10^{-6}, \\
D_{h}=-2 \cdot 10^{-6}, D_{h}=-7 \cdot 10^{-7} .
\end{gathered}
$$

The first two values $D_{h}$ are small and the rest are below the accuracy accepted in calculation of the data in the Table 1 . Therefore, we can put $D_{h} \approx 0$ for calculation of the approximate values $h$ by all regions of $B_{1}$, where the solution does not exist because $1+2 a_{1} A+2 a_{2} A^{2}=0, \quad h=h_{1,2}=$ $\left(a_{22}-a_{11} a_{12}\right) / a_{12}^{2}$. Thus, the approximations of the profiles are unattainable; they do not satisfy (20).

The functions $h_{0}\left(i_{0}\right), h_{i}\left(i_{0}\right)$ in (19) have the breaks at the transition points of the permanent function $B_{1}^{(n)}(\eta, h)$ from the one regional approximation to the other one (a first derivative has break at those points). It is impossible to get a common approximation for $B_{l}(\eta, h)$ satisfying the boundary conditions in all range by $i_{0}$ (due to variation of $B_{l}$ between 0 and 1 ).

The calculations revealed no substantial difference in the final results of numerical solution at the transformation points of the functions $B_{l}(\eta, h)$. Some results by estimation of the working regions for the function $h$ depending on the parameter $i_{0}$ are presented below in Figs 7-11.

For the first approximation of the functionindicator of the first phase, $B_{1}^{(0)}$, it is computed the initial value of the parameter $h$, which is $h_{0}=0$. The corresponding value $i_{0} \approx 15.9$; for the end of the initial part of the jet it is, correspondingly $h_{i}=0$, $i_{0} \approx 20.4$. Therefore, the working region is by $h_{0}$ and $h_{i}: i_{0} \in[1.3 ; 15.9]$ and $i_{0} \in[1.8 ; 20.4]$, respectively. Thus, common region is $i_{0} \in[1.8 ; 15.9]$ - for mixing of the liquids when the denser is surrounding one.

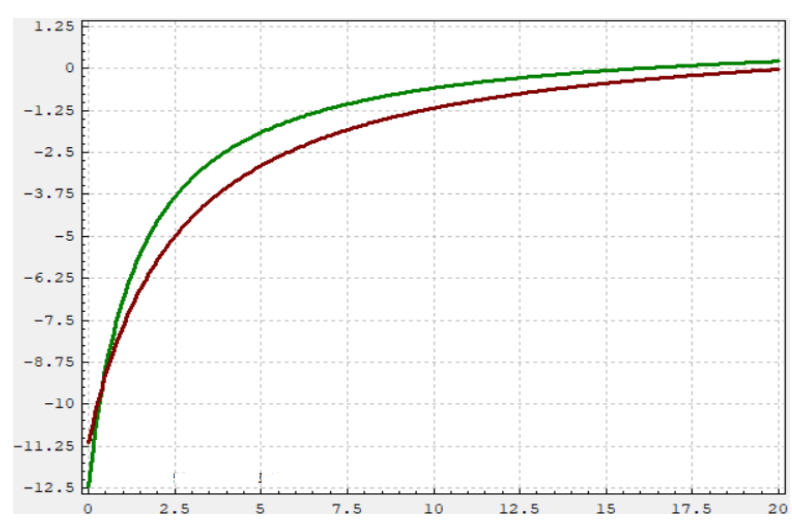

Fig. 7 Functions $h_{0}$ (up) and $h_{i}$ (down) versus parameter $i_{0}$ for the region $h \in[-6,0]$

Because $i_{0}=n s_{0}^{2}$, this region fits for nearly all cases starting from equal densities of the mixing liquids $(n=1)$ because a slip of phases is influencing as quadrate $s_{0}^{2}$ so that even comparably small slip $s_{0}=0.8$ gives respectively $\rho_{2} / \rho_{1}=1.8 / 0.64=2.8$, $\rho_{2} / \rho_{1}=15.9 / 0.64=24.8$. Thus, $\rho_{2} / \rho_{1} \in[2 ; 20]$ supposed to be approximately, where the last limit value of the density ratio may correspond to vapourliquid or similar mixing liquids.

Then similarly calculation for the next approximation of the function-indicator, $B_{1}^{(1)}$, results in $h_{0}=0, i_{0} \approx 4.07, h_{i}=0, i_{0} \approx 5.2$. Here the working region is $h \in[-12,-6]$ by $h_{0}$ and $h_{i}$, respectively: $i_{0} \in[0.5 ; 1.25]$ and $i_{0} \in[0.84 ; 3.5]$.

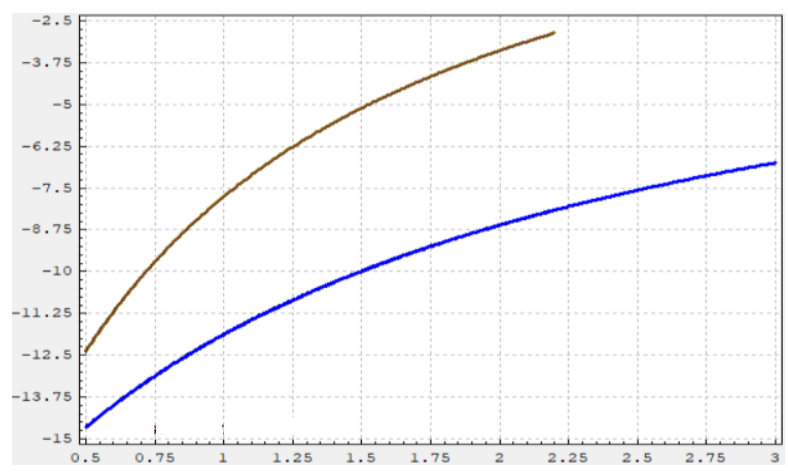

Fig. 8 Functions $h_{0}$ (up) and $h_{i}$ (down) versus parameter $i_{0}$ for the region $h \in[-12,-6]$ 
The common region is $i_{0} \in[0.84 ; 1.25]$, which is narrower than the previous one but probably the most useful in practice. It might be something like $\rho_{2} / \rho_{1} \in[1 ; 1.5]$.

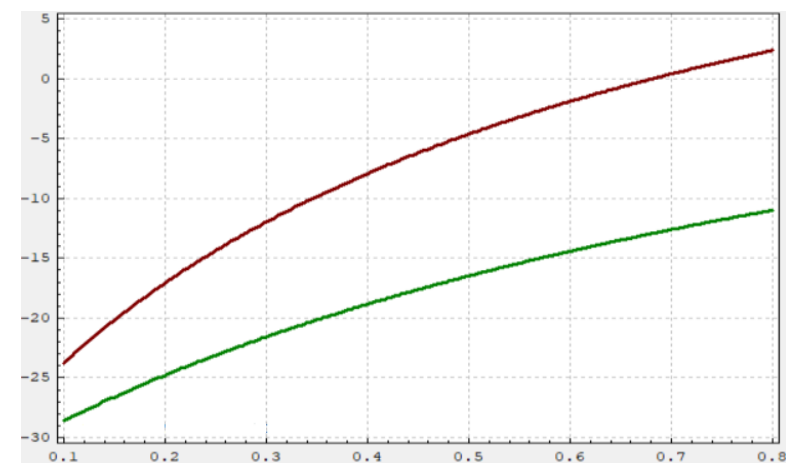

Fig. 9 Functions $h_{0}$ (up) and $h_{i}$ (down) versus parameter $i_{0}$ for the region $h \in[-20,-12]$

For the third approximation region of the function-indicator according to (5), $B_{1}^{(2)}$, the beginning and the end of the initial part of the jet have the following parameters: $h_{0}=0, i_{0} \approx 0.68$, $h_{i}=0, i_{0} \approx 2.03$. The working region $h \in[-20,-12]$ is by $h_{0}$ and $h_{i}$, respectively, for: $i_{0} \in[0.16 ; 0.3]$ and $i_{0} \in[0.35 ; 0.63]$. Thus, the common region by parameter $i_{0}$ for the beginning and for the end of the initial part of the jet does not exist here, except approximately one value $i_{0}=0.3$, which may be considered as the same because $i_{0}=0.3$ and $i_{0}=0.35$ are close and a slip progressing of the phases during flow in a mixing zone is available.

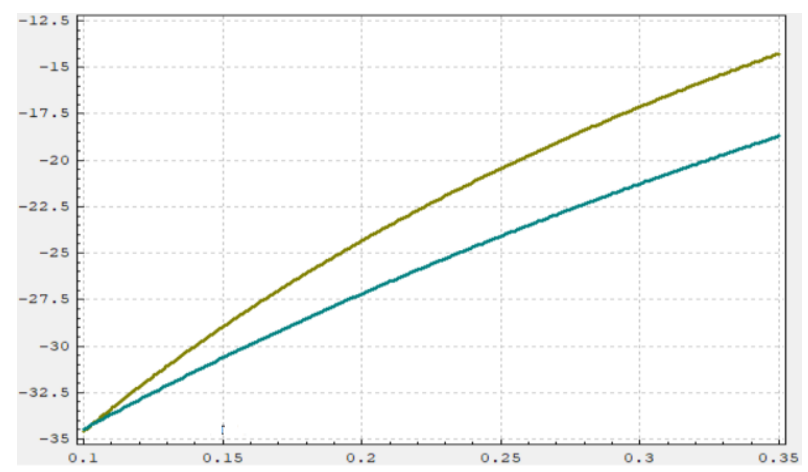

Fig. 10 Functions $h_{0}$ (up) and $h_{i}$ (down) versus parameter $i_{0}$ for the region $h \in[-30,-20]$

Then for the $B_{1}^{(3)}$ follows $h \in[-30,-20], h_{0}=0$, $i_{0} \approx 0.77, h_{i}=0, i_{0} \approx 0.93$ - working region is by $h_{0}$ and $h_{i}: i_{0} \in[0.14 ; 0.26]$ and $i_{0} \in[0.16 ; 0.33]$. The common region for the beginning and for the end of initial part is $i_{0} \in[0.16 ; 0.26]$, narrow, for a small $\rho_{2} / \rho_{1}$. And finally, $B_{1}^{(4)}, h \in[-42,-30], h_{0}=0$, $i_{0} \approx 0.42, h_{i}=0, i_{0} \approx 0.49$. Working region is by $h_{0}$, $h_{i}$, respectively: $i_{0} \in[0.09 ; 0.15]$ and $i_{0} \in[0.1 ; 0.17]$. The common region by $i_{0}$ for the beginning and for the end of initial part is $i_{0} \in[0.1 ; 0.17]$, very narrow, for a very small density ratio $\rho_{2} / \rho_{1}$ (e.g. like a spreading of a hot liquid in saturated vapour).

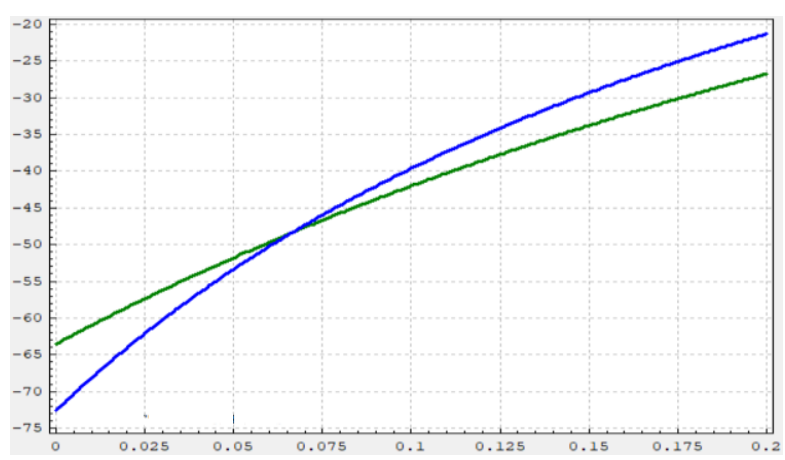

Fig. 11 Functions $h_{0}$ (up) and $h_{i}$ (down) versus parameter $i_{0}$ for the region $h \in[-42,-30]$

\subsection{Numerical Simulation of Initial Jet' Part}

Substitution of $h_{i}\left(i_{0}\right)$ into (18) results in correlation for the radius of a jet at the end of initial part, $\delta_{i}\left(i_{0}\right)$. The (18) determine functions $y_{0}(h), \delta(h)$ in the range $h \in\left[h_{0}, h_{i}\right]$, which are monotonous and close to the linear ones by $i_{0}$ over 5 , rapidly falling by $i_{0}$ below 1 .

Differential equation from the system (10) is solved numerically with account of the solution (18). This equation is written for the part of the mixing zone, we use it for the half of mixing layer: $\eta=\eta^{*}=0.5$. Equation array for $y_{0}(\varsigma), h(\varsigma)$ is:

$$
\begin{aligned}
& \frac{d h}{d \varsigma}=-\frac{1}{y_{0}^{3} D_{2}} \cdot \frac{d y_{0}}{d \varsigma}, \frac{d y_{0}}{d \varsigma}=y_{0}^{2} D_{2}\left(1+A \eta^{*}\right) . \\
& \cdot \frac{B_{1}^{*}\left(\partial u_{1} / \partial \eta\right)^{*}+i_{0} \kappa_{21} B_{2}^{*}\left(\partial u_{2} / \partial \eta\right)^{*}}{\left(1-u_{1}^{*}+2 D_{1}\right) y_{0}^{2} D_{2}-d D_{1} / d h} .
\end{aligned}
$$

Equation array (21) is solved numerically with the initial condition (12), (19), where the expression $h_{0}=h(0)$ is the initial condition for (21), while (18) and $h_{i}=h\left(\varsigma_{i}\right)$ are used for control of the numerical solution. Here are the following parameters: 


$$
\begin{gathered}
\left(\partial u_{2} / \partial \eta\right)^{*}=\left(\partial u_{1} / \partial \eta\right)^{*}=-1.5, B_{1}^{*}=B_{1}\left(\eta^{*}\right), \\
\partial u_{1} / \partial \eta=12 \eta^{2}(\eta-1), \partial u_{2} / \partial \eta=-12 \eta(\eta-1)^{2}, \\
u_{1}^{*}=11 / 16, u_{2}^{*}=5 / 16, A=\frac{a_{3}+i_{0} b_{3}-a_{1}}{a_{2}-i_{0} b_{4}-a_{4}}, \\
D_{1}=A\left[a_{3}^{*}+i_{0} b_{3}^{*}-a_{1}^{*} u_{1}^{*}-i_{0} b_{1}^{*} u_{2}^{*}+\right. \\
\left.+A\left(a_{4}^{*}+i_{0} b_{4}^{*}-a_{2}^{*} u_{1}^{*}-i_{0} b_{2}^{*} u_{2}^{*}\right)\right\rfloor \\
\frac{d A}{d h}=\frac{a_{32}+i_{0} b_{32}-a_{12}}{a_{2}-i_{0} b_{4}-a_{4}}-A \frac{a_{22}-i_{0} b_{42}-a_{42}}{a_{2}-i_{0} b_{4}-a_{4}} \\
D_{2}=a_{22} A^{2}+A\left(a_{12}+2 a_{2} \frac{d A}{d h}\right)+a_{1} \frac{d A}{d h} \\
\frac{d D_{1}}{d h}=\frac{d A}{d h}\left(a_{3}^{*}+i_{0} b_{3}^{*}-a_{1}^{*} u_{1}^{*}-i_{0} b_{1}^{*} u_{2}^{*}\right)+ \\
A\left(a_{32}^{*}+i_{0} b_{32}^{*}-a_{12}^{*} u_{1}^{*}-i_{0} b_{12}^{*} u_{2}^{*}\right)+ \\
+2 A \frac{d A}{d h}\left(a_{4}^{*}+i_{0} b_{4}^{*}-a_{2}^{*} u_{1}^{*}-i_{0} b_{2}^{*} u_{2}^{*}\right)+ \\
A^{2}\left(a_{42}^{*}+i_{0} b_{42}^{*}-a_{22}^{*} u_{1}^{*}-i_{0} b_{22}^{*} u_{2}^{*}\right)
\end{gathered}
$$

\subsection{Numerical Simulation of Ground Part}

From the first two equations (14) for the ground part of a jet with the boundary conditions (16) yields

$$
\begin{aligned}
h & =\frac{\alpha_{11}-\left(\alpha_{21}+i_{0} \beta_{21}\right) u_{m 1}-2 i_{0} \alpha_{11} \beta_{20} u_{m 1}^{2} \delta^{2}}{-\alpha_{12}+\left(\alpha_{22}+i_{0} \beta_{21}\right) u_{m 1}+2 i_{0} \alpha_{12} \beta_{20} u_{m 1}^{2} \delta^{2}}, \\
B_{m 1} & =\frac{2 i_{0}}{Z} \alpha_{12} \beta_{20}+\frac{\alpha_{22}+i_{0} \beta_{22}}{Z u_{m 1} \delta^{2}}-\frac{\alpha_{12}}{Z u_{m 1}^{2} \delta^{2}},
\end{aligned}
$$

The condition $\zeta=\infty, B_{m l}=0$ leads the following: $\zeta=$ $\infty, u_{m l} \delta^{2}=\infty, u_{m l} \delta=$ const, so that follows:

$$
\lim _{\zeta \rightarrow \infty} \frac{\alpha_{12}}{Z}\left(2 i_{0} \beta_{20}-\frac{1}{u_{m 1}^{2} \delta^{2}}\right)=0,
$$

where from with account of $\propto_{12} \neq 0$ yields

$$
\begin{gathered}
\lim _{\zeta \rightarrow \infty} u_{m 1}^{2} \delta^{2}=\frac{1}{2 i_{0} \beta_{20}} \approx\left\{\begin{array}{ll}
33, & i_{0}=0.3 \\
10, & i_{0}=1.0 \\
1.2, & i_{0}=8.0
\end{array},\right. \\
h_{\infty \rightarrow \infty}=\lim h \approx\left\{\begin{array}{ll}
-70, & i_{0}=0.3 \\
-31, & i_{0}=1.0 . \\
-18, & i_{0}=8.0
\end{array},\right.
\end{gathered}
$$

The value $h_{\infty}$ is outside the region of the function $h$ variation determined by $h=h_{t}$ (beginning of the ground part). Therefore, function $B_{1}$ is continuous for each value $i_{0}$ (its first derivative is piecewise continuous). It is changing its approximation with transformation of the region by $h$.

\section{The conclusions}

Mathematical model obtained allowed studying the important features of the turbulent two-phase jets of immiscible liquids. The physical phenomena and peculiarities of the model were described in detail. The model is applicable for detail study of turbulent two-phase flows and heat transfer.

As shown in the paper the limitation of the model for turbulent heterogeneous jets of immiscible liquids is in touch with polynomial approximations of the function-indicator of the phases. Despite the proposed simple algorithm for control of the transformation of approximations from one to another region according to the varying parameters, under certain conditions, it is impossible to compute the whole jet flow in the frame of one approximation for one set of parameters. Also it was mentioned about available numerical singularities in solution of the boundary problems and shown the ways to avoid them.

The directions of future research are suggested to be for improvements of the approximations of the profiles and function-indicator based on the results of the experimental study of the phase distribution in multiphase flows using the special two-phase sensors.

\section{References:}

1. Kazachkov I.V. Parametric Excitation and Suppression of Oscillations at the Interfaces of Continua for the Processes' Control in Jet and Film Flows, Channel Flows with Phase Change and in Granular Media// J. of Theoretical and Applied Mechanics// 2015, 10, P. 1-23 .

2. Kazachkov I.V., Kamaev Yu.M., Khutornyi V.M., Tomyak R.Ya. Modeling of the Thermal Processes in Repository of the Waste Nuclear Fuel with Water for Assessment of Potentially Hazardous Situations// WSEAS Transactions on Heat and Mass Transfer, 2019, 14(17), p. 137146.

3. Hetsroni G., Sokolov M. Distribution of mass, velocity and intensity of turbulence in a twophase turbulent jet// Trans. ASME J. Appl. Mech., 1971, 38(2), 315.

4. Deich M.E., Filippov G.A. Gazodinamika dvukhfaznykh sred (The Gas Dynamics of TwoPhase Media). Moscow: Energoizdat, 1981, 472 pp.

5. Ram Sinha. Turbulent transport coefficients for compressible heterogeneous mixing// Int. J. of 
Heat and Mass Transfer, 1973, vol. 16, 5, p. 1048-1052.

6. Baranovskii S.I. Characteristic features of highvelocity two-phase gas-liquid flows. Turbulentnye dvukhfaznye techeniya i tekhnika eksperimenta (Turbulent Two-Phase Flows and Experimental Techniques), Tallinn, 1985, p. 60.

7. Lepeshinskii I.A., Sovetov V.A., Chabanov V.A. A model of turbulent interaction of the phases of a multiphase multicomponent nonisothermal nonequilibrium jet. In: Turbulentnye dvukhfaznye techeniya i tekhnika eksperimenta (Turbulent Two-Phase Flows and Experimental Techniques), Tallinn, 1985, Part 2, p. 42.

8. Clayton T. Crowe, John D. Schwarzkopf, Martin Sommerfeld, Yutaka Tsuj. Multiphase Flows with Droplets and Particles, CRC, Boca Raton, 2011, 509 pp.

9. Modarress D., Tan H., Elghobashi S. Twocomponent LDA measurement in a two-phase turbulent jet// AIAA J., 1984, 22(5), 624.

10. Elghobashi S. Particle-Laden turbulent flows: Direct simulation and closure models// Appl. Sci. Res., 1991, 48, 301.

11. Gore R.A., Crowe C.T.: Modulation of turbulence by a dispersed phase// Trans. ASME J. Fluids Eng., 1991, 113(2), 304.

12. Ahmed A.M., Elghobashi S. On the mechanisms of modifying the structure of turbulent homogeneous shear flows by dispersed particles// Phys. Fluid, 2000, 12, 2906.

13. Emil Sekula. The structure of turbulent jets: application of experimental and environmental methods. Barcelona: 2010, 229 p.

14. Kuang C.P., Lee J.H.W. Stability and Mixing of a Vertical Axisymmetric Buoyant Jet in Shallow Water// Environmental Fluid Mechanics. 2006, 6: p. 153-180.

15. Khodadadi J.M. and Vlachost N.S. Experimental and Numerical Study of Confined Coaxial Turbulent Jets// AIAA Journal, 1989, vol. 27, No. 5.

16. Dimotakis P.E. The mixing transition in turbulent flows// J. Fluid Mechanics, 2000, vol. 409, p. 69-98.

17. Angela Hilgers. Control and optimization of turbulent jet mixing. Center for Turbulence Research Annual Research Briefs 2000.

18. Agrawal A., Prasad A.K. Integral Solution for the Mean Flow Profiles of Turbulent Jets, Plumes and Wakes// Journal of fluids engineering, 2003, vol. 125(5), p. 813822.
19. Klazly M.M., and Bognár G., CFD Study for the Flow Behaviour of Nanofluid Flow over Flat Plate// Int. J. of Mechanics, 2020, Vol. 14, p. 49-57.

20. Manuel A. Falconi, Enrique T. Tamayo, Héctor L. Laurencio, Jorge P. Vega, Elvis P. Gualotuña, Edwin R. Grijalva, Luis Grijalva Campana, Model of pressure losses in pipes during the transport of heavy oil with 11 API gravity// Int. J. of Mechanics, 2018, Vol. 12, p. 8-13.

21. Nakorchevski A.I. Reactor for continuous refinement of metal/ Patent USSR №492554.Bulletin.- 1975.- №43.

22. Nakorchevskii A.I. Heterogeneous turbulent jets. Kyiv: Naukova Dumka, 1980, 142 p.

23. Nakorchevskii A.I., Kazachkov I.V. Calculation of the heterogeneous turbulent jet. In book: Systems of automation of continuous technological processes, Institute of Cybernetics of NASU, 1979, P. 68-79.

24. Kazachkov I.V. Mathematical modelling of heterogeneous turbulent jets in cylindrical chamber// Soviet automatic control, 1980, vol.13, jan.-feb., p. 1-6.

25. Kazachkov I.V. Approaches for Mathematical Modeling and Experimental Study of the Turbulent Flows of Mutually Immiscible Liquids (Oil-Water)// Int. J. Petroleum and Petrochem. Engineering (IJPPE), 2018, 4(1), p. 70-81.

26. Nakorchevskii A.I., Martynenko M.P., Basok B.I. Calculation of Chamber-Type Pulsers// Journal of Engineering Physics and Thermophysics, 2004, 77, p. 156-160.

27. Nakorchevskii A.I. Action of climate condition on heat transfer through the fencing construction of buildings and use of excess heat for their heating// Journal of Engineering Physics and Thermo-physics. 2013, 86(3), p. 229-241.

28. Nakorchevskii A.I., Basok B.I. Hydrodynamics and heat transfer in heterogeneous systems and devices of pulsating type. Kyiv: Naukova Dumka, 2001, 348 p.

29. Nakoryakov V.E., Pokusaev B.G., Shreiber I.R. Spreading of the waves in gas- and vapor-liquid media. Novosibirsk: ITF, 1983, $238 \mathrm{p}$.

30. Nigmatulin R.I. and Friedly J.C. Dynamics of Multiphase Media. CRC Press: Volumes $1 \& 2$. Revised and Augmented Edition, Oct 1, 1990, $878 \mathrm{p}$.

31. Ginevskii A.S. The theory of turbulent jets and traces. Integral calculation methods. Moscow: Mashinostroenie, 1969, $400 \mathrm{p}$. 


\section{Creative Commons Attribution License 4.0} (Attribution 4.0 International, CC BY 4.0)

This article is published under the terms of the Creative Commons Attribution License 4.0

https://creativecommons.org/licenses/by/4.0/deed.en US 